\title{
The architecture of weighted mutualistic networks
}

\author{
Luis J. Gilarranz, Juan M. Pastor and Javier Galeano \\ L.J. Gilarranz (lj.gilarranz@ebd.csic.es), Estación Biológica de Doñana, CSIC, C/Américo Vespucio s/n, ES-41092 Sevilla, Spain. \\ -J. M. Pastor and J. Galeano, Dept. Ciencia y Tecnología Aplicadas a la I. T. Agrícola, EUIT Agrícola, Univ. Politécnica de Madrid, \\ Ciudad Universitaria s/n, ES-28040 Madrid, Spain.
}

\begin{abstract}
Several ecosystem services directly depend on mutualistic interactions. In species rich communities, these interactions can be studied using network theory. Current knowledge of mutualistic networks is based mainly on binary links; however, little is known about the role played by the weights of the interactions between species. What new information can be extracted by analyzing weighted mutualistic networks? In performing an exhaustive analysis of the topological properties of 29 weighted mutualistic networks, our results show that the generalist species, defined as those with a larger number of interactions in a network, also have the strongest interactions. Though most interactions of generalists are with specialists, the strongest interactions occur between generalists. As a result and by defining binary and weighted clustering coefficients for bipartite networks, we demonstrate that generalists form strongly-interconnected groups of species. The existence of these strong clusters reinforces the idea that generalist species govern the coevolution of the whole community.
\end{abstract}

In the past few years, several studies have used complex network theory to unveil hidden secrets of nature. The patterns of interactions between species provide information about the stability of ecological communities (Solé and Montoya 2001, Rezende et al. 2007, Stouffer and Bascompte 2010), give a clue to understand their diversity (Bastolla et al. 2009), help us to assess ecosystem services (Dobson et al. 2006), and enlighten us about the coevolutionary process (Thompson 2006). Further understanding of the architecture of complex ecological networks will shed more light onto these questions.

One kind of ecological network, mutualistic networks, depicts the interactions of mutual benefit between species in a community. Here, a set of species A, generally animals, interacts with another set of species P, generally plants, but there are no interactions within sets. Such a network can also be referred to as a bipartite network. Species are represented as nodes, and the interactions between these species are represented by links. The information about interactions can be binary, where a link indicates just an interaction between two species, or can be weighted, where the weight represents how strong that interaction is (Bascompte and Jordano 2007).

While the aforementioned works have analyzed the structural properties of mutualistic networks in their binary representation, a smaller but growing number of papers have analyzed the relevance of interaction weights. A seminal work by Bascompte and coworkers (Bascompte et al. 2006), found that the asymmetry of dependences (Jordano 1987) between species in an interaction allows for greater biodiversity. Scotti et al. (2007), analyzed how the rank of species importance, based on their position in the network, changes when considering interaction weights. Though the ranks where very different in food webs, they found smaller differences in plant-pollinator networks. Blüthgen et al. (2008), apply information theory measures to characterize the degree of specialization in quantitative mutualistic networks. Hwang et al. (2009), showed that species strength, defined as species' total number of visits, is correlated with species degree in six plant-pollinator networks. Despite these first studies, the complete structure of weighted mutualistic networks, and how interaction weights are related to network structure, is still poorly known.

For the majority of mutualistic networks, interaction weights are usually measured as the number of recorded visits (Supplementary material Appendix 1 Table A1). Other interaction weight measures include the number of individuals caught, the frequency of visits, or the number of fruits removed in dispersal networks. It has been demonstrated that pollinators differ in their pollination effectiveness qualitatively (Herrera 1987) and quantitatively (Herrera 1989), as do the same for seed dispersers (Schupp et al. 2010). Nevertheless, Vazquez et al. (2005) showed that interaction frequency can be used as a surrogate for the total effect of one species on another in a mutualistic community. With this in mind, we expect that the patterns from weighted networks described here will not qualitatively change when considering different measures of interaction weight.

Let us first describe traditional network characteristics for unweighted mutualistic networks. The standard way to characterize a species is by its degree, which is defined as its number of interactions with other species. Degree provides 
a first hint about the species' functional role in the network. Although measuring specialization in the field may be challenging, in the grounds of both ecological (Waser and Ollerton 2006) and coevolutionary (Thompson 2005) theory, specialization is here defined as the number of species a focal species interacts with. This widely used definition establishes a gradient between generalist and specialist species. Therefore, the most connected species are generalists whereas specialists have few interactions. At the network level, a first characterization of network structure is given by the degree distribution. This is the frequency distribution of the number of links, or degree, per species. Jordano and coworkers studied the degree distributions of 29 plantpollinator and 24 plant-frugivore networks (Jordano et al. 2003). Most of the networks exhibited degree distributions with a truncated power-law regime. This means that most species have very few links while few species have many more interactions than would be expected by random networks with similar properties.

Not all interactions, however, are equally strong. That is to say, species with several interactions will have interactions of varying weights. The unweighted network, therefore, may not tell the whole story (Jordano 1987). In a weighted network, the sum of the weights of all interactions of a species gives the species' strength. Strength is the weighted analog to species' degree, and is a measure of the centrality of a species in the network. Species centrality is correlated with the species' pattern of interaction and its functional role in a community or Eltonian niche (Elton 1927). The strength of an species i, si, was defined by Barrat et al. (2004) as

$s_{i}=\sum_{j=1}^{N} a_{i j} w_{i j}$

where $\mathrm{a}$ is the adjacency matrix whose elements $\mathrm{a}_{\mathrm{ij}}$ take the value 1 if a link connects species $i$ to the species $j$ and 0 otherwise. The weight of the every link is given by the matrix $\mathrm{w}$ and $\mathrm{N}$ is the total number of nodes. In this paper, we have used the literal definition of Barrat and coworkers, where the weight $\mathrm{w}_{\mathrm{ij}}$ of a link represents the number of the visits between these species, being $\mathrm{w}_{\mathrm{ij}}=\mathrm{w}_{\mathrm{ji}}$. Nevertheless Bascompte et al. (2006) defined a similar species strength as the sum of the dependences, $d$, of all animals on a specific plant or the sum of dependences of all plants on a specific animal. They found the dependences matrix to be asymmetric, being $d_{i j}$ different from $\mathrm{d}_{\mathrm{ji}}$.

A node's degree and strength are measures that provide information about the centrality of a single species within a network structure. Previous studies traced a relationship between node's degree and the relevance of that node to the structural stability of the network (Solé and Montoya 2001). Species in a network can be ranked according to this criterion of node relevance (Scotti et al. 2007), this sort of relevance is known as node's centrality. Throughout the paper, we explore some characteristics of species within their communities that are plotted as a function of node's degree. We can therefore study how some species properties change according to their relevance for community stability.

Here, we ask the question of what new information can be extracted by analyzing weighted mutualistic networks. We develop and compare equivalent metrics in unweighted and weighted networks and examine to what extent the observed patterns change and why. What new questions can be addressed by using quantitative networks? How do our results complement previous work? To answer these questions, we examine a set of statistical measures (Barrat et al. 2004), such as: distribution of weights, strength-degree correlation, average weight as a function of end-point degree, and average nearest-neighbors' degree. Furthermore, we introduce two new clustering measures for binary and weighted bipartite networks that provide novel information about whether species tend to be organized in small interconnected groups.

\section{Material and methods}

We have analyzed the topological properties of twenty nine weighted mutualistic networks. Twenty of them represent interactions between plants and pollinators, six represent interactions between plants and seed dispersers, and three networks represent interactions between ants and trees (Supplementary material Table A1). With these networks, we have calculated several metrics commonly used in complex networks analysis. We then quantified the structure of the mutualistic networks taking into account the weight of the interactions. This allows us to answer several questions, as detailed in the following.

1) What is the relationship between species' strength and its degree? To answer this question we plot the average strength of species with the same degree as a function of species degree, k. Barrat et al. (2004) showed that this relationship is well fit by the curve:

$\mathrm{s}(\mathrm{k}) \sim \mathrm{k}^{\beta}$

Since species' degree, $\mathrm{k}$, and species strength, s, are correlated, but not necessarily linearly, the parameter $\beta$ provides information about the shape of this correlation. One possibility is that those species with a larger degree have many interactions but with low weights and thus a low strength. In these networks, the importance of highly-connected species would be lower than that predicted by its degree. This behavior would correspond to $\beta<1$. Another possibility is that interaction weights are randomly distributed. In this case, corresponding to $\beta \approx 1$, degree and strength exhibit a linear correlation and both measures provide the same information about species' importance. In this null case, we can approximate interaction weight between every two species, $\mathrm{w}_{\mathrm{ij}}$, as the average interaction weight across the community. The third possibility is that highly-connected species tend to have stronger interactions than the average interaction weight. In this case, species' degree and species' strength are not linearly correlated, and just considering species' degree would underestimate the importance of the most-connected species and overestimate the importance of less connected species. This third case corresponds to $\beta>1$.

2) Are links between generalists stronger than links between specialists? Not all interactions from a species have the same weight. Species exhibit preferences for some of the other species that they are connected to. How do species tend to allocate their visits? One way to answer this question is to calculate the dependence of the weight, $\mathrm{w}_{\mathrm{ij}}$, on the 
end-point degree of a link (Barrat et al. 2004). End-point degree of a link is the product $\mathrm{k}_{\mathrm{i}} \cdot \mathrm{k}_{\mathrm{j}}$ of the degrees of the two nodes connected by that link. Barrat and coworkers shown that the average weight as a function of end-point degree can be approximated in some cases as

$$
\mathrm{w}\left(\mathrm{k}_{\mathrm{i}} \times \mathrm{k}_{\mathrm{j}}\right) \sim\left(\mathrm{k}_{\mathrm{i}} \times \mathrm{k}_{\mathrm{j}}\right)^{\theta}
$$

where $\mathrm{w}\left(\mathrm{k}_{\mathrm{i}} \times \mathrm{k}_{\mathrm{j}}\right)$ represents the average weight of links with a certain end point degree. If the exponent $\theta$ is greater than zero, there is a positive correlation between end-point degree and the average weight of a link. In this case, highlyconnected nodes tend to be linked via strong interactions. If the exponent $\theta$ is below zero, the opposite occurs.

These two previous measures provide extra information about the importance of the node in the network, information that can be only obtained from weighted networks. To quantify the structural organization of the network, we can explore other measures such as the clustering coefficient. The clustering coefficient of a species in unipartite networks such as food webs, where all nodes are of the same type and links are allowed between any two nodes, measures the fraction of interconnected neighbors of a given node, i.e. the fraction of species, connected to a focal species, that are connected themselves. Networks with a large clustering coefficient, and with a small average number of links between any two nodes in the network are referred to as 'small worlds' (Watts and Strogatz 1998). Mutualistic networks have small world properties (Olesen et al. 2006). Therefore, what affects one species will indirectly affect all species to which it is connected and so on. The clustering coefficient can thus have large implications for how fast a perturbation propagates across the network (Pastor-Satorras and Vespignani 2001).

Since mutualistic networks are bipartite graphs, plants linked to an animal cannot interact with other plants, and vice versa. Unfortunately, the traditional clustering coefficient is therefore always zero. To get around this, the standard way to calculate the clustering coefficient is to project (a)

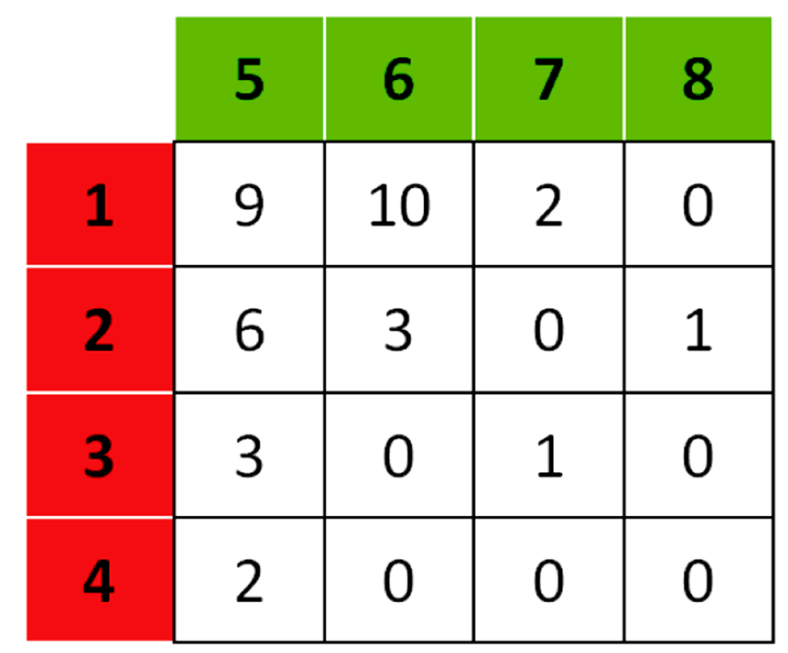

(b) Graph representation

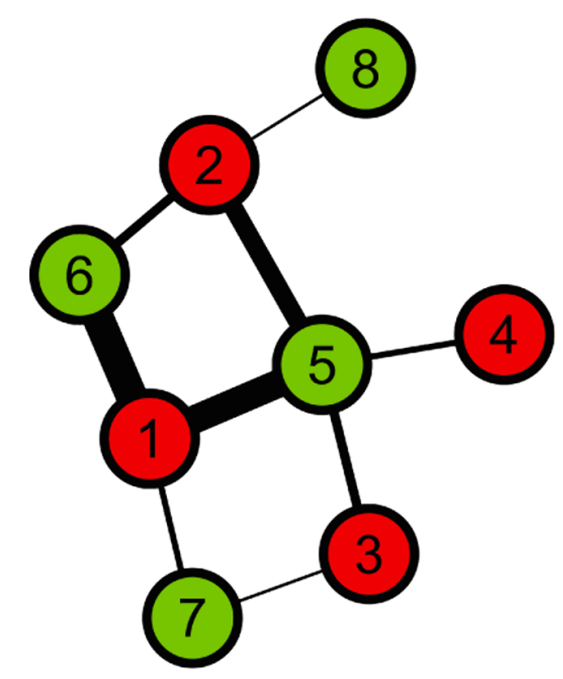

(c)

$$
\text { Unweighted } \begin{aligned}
C_{4 b}(1) & =\frac{q_{1}}{k_{1}^{n n} \times k_{1}\left(k_{1}-1\right) / 2}=\frac{2}{3 \times 3(3-1) / 2}=\frac{2}{9}=0.222 \\
\text { Weighted } \quad C_{4 b}^{w}(1) & =\frac{\left[q_{1,5,7}\left(\frac{\frac{w_{1,5}}{s_{1} / k_{1}}}{2}+\frac{\frac{w_{1,7}}{s_{1} / k_{1}}}{2}\right)\right]+\left[q_{1,6,5}\left(\frac{\frac{w_{1,6}}{s_{1} / k_{1}}}{2}+\frac{\frac{w_{1,5}}{s_{1} / k_{1}}}{2}\right)\right]}{k_{1}^{n n} \times k_{1}\left(k_{1}-1\right) / 2} \\
& =\frac{1 \times \frac{\frac{9}{21 / 3}}{2}+\frac{\frac{2}{21 / 3}}{2}+1 \times \frac{\frac{10}{21 / 3}}{2}+\frac{\frac{9}{21 / 3}}{2}}{3 \times 3(3-1) / 2}=0.238
\end{aligned}
$$

Figure 1. Schematic illustration of how to compute unweighted and weighted bipartite clustering coefficient following Eq. 5 and Eq. 6. (a) The example graph is represented as a matrix w, where 0 represents no interaction and non-zero values indicate the weight of the link between two species. In the matrix representation, animals appear in rows and plants in columns. The colored boxes contain the nodes' labels, red for animals and green for plants. (b) The network can also be represented as a graph, where species are linked by lines with their width proportional to link weight. (c) In this example, we calculate both clustering coefficients for the species labeled $\mathrm{i}=1$, which is the animal corresponding to the first row of $\mathrm{w}$ matrix, with $\mathrm{k}_{1}=3 ; \mathrm{s}_{1}=21 ; \mathrm{q}_{1}=2 ; \mathrm{k}_{1}^{\mathrm{nn}}=3$. It takes part in two quadrilaterals formed by nodes (1-6-2-5) and (1-7-3-5), respectively. In the example, the weighted clustering coefficient is greater than the unweighted clustering coefficient. 

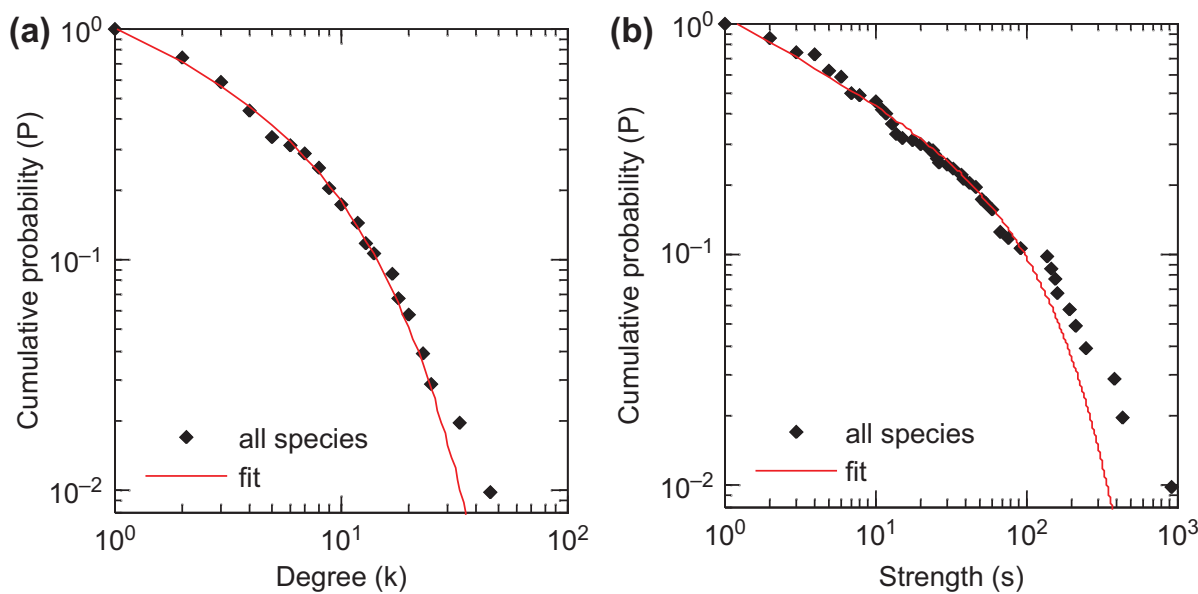

Figure 2. (a) Cumulative probability distribution of node degree for all species. The solid line is the fit to a truncated power-law $\mathrm{P}(\mathrm{k}) \approx \mathrm{k}^{-\gamma}$ $\exp \left(-\mathrm{k} / \mathrm{k}_{\mathrm{c}}\right)$ with parameters $\gamma=0.35 \pm 0.03$ and $\mathrm{k}_{\mathrm{c}}=9.7 \pm 0.9$. (b) Cumulative probability distribution of node strength for all species. The solid line is the fit to a truncated power-law $\mathrm{P}(\mathrm{s}) \approx \mathrm{s}^{-\gamma} \exp \left(-\mathrm{s} / \mathrm{s}_{\mathrm{c}}\right)$ with parameters $\gamma=0.36 \pm 0.02$ and $\mathrm{s}_{\mathrm{c}}=134 \pm 23$.

a bipartite graph into two graphs (Onody and de Castro 2004, Olesen et al. 2006). The first graph is for animals; two animals are connected if they share a plant in the bipartite graph. The second graph is for plants following the same procedure. Traditional clustering coefficient analyses can then be performed. This projection procedure is not without its problems, however. The original network structure is lost, and the meaning of links is changed. In plant-pollinator networks, for example, links in the bipartite network represent pollination interactions, but in the projected networks a link between two species represents niche overlap (Ribeiro Mello et al. 2011).

Another option that does not change the original structure of the graph or links' meaning, is to calculate the clustering coefficient for cycles of four connections, $\mathrm{C}_{4}$, as suggested by Lind et al. (2005). In order to adapt their procedure to mutualistic networks, we propose a specific definition of clustering coefficient for bipartite graphs, $\mathrm{C}_{4 \mathrm{~b}}$, where interactions inside a set don't exist. We call all species that are connected to a species $i$ that species' first neighbors. The second neighbors of species $i$ are then all species connected to their first neighbors but that are not directly connected to i. The bipartite clustering coefficient for an species i can be defined as the fraction of common connections between second-nearest neighbors (not counting i). If a second-nearest neighbor is connected to two neighbors of node $\mathrm{i}$, then one can find a quadrilateral formed by node $i$, by the two first neighbors that share a neighbor different from $i$ (that node is a second-nearest neighbor of $i$ ), and by the species shared by the two first neighbors of i. In Fig. 1, we illustrate a case study to visually help to the calculation of $\mathrm{C}_{4 \mathrm{~b}}(\mathrm{i})$. The bipartite clustering coefficient $\mathrm{C}_{4 \mathrm{~b}}(\mathrm{i})$ for node $\mathrm{i}$ is defined as

$\mathrm{C}_{4 \mathrm{~b}}(\mathrm{i})=\frac{\mathrm{q}_{\mathrm{i}}}{\mathrm{Q}_{\mathrm{i}}}$

where $q_{i}$ is the number of observed quadrilaterals around node $i$ and $Q_{i}$ is the total number of possible quadrilaterals around node $i$. The total number of possible quadrilaterals is the number of second-nearest neighbors, $\mathrm{k}_{\mathrm{i}}^{\mathrm{nn}}$, multiplied by the total number of pairs of neighbors given by $\mathrm{k}_{\mathrm{i}}\left(\mathrm{k}_{\mathrm{i}}-1\right) / 2$. The bipartite clustering coefficient can then be defined as

$$
\mathrm{C}_{4 \mathrm{~b}}(\mathrm{i})=\frac{\mathrm{q}_{\mathrm{i}}}{\mathrm{k}_{\mathrm{i}}^{\mathrm{nn}} \times \mathrm{k}_{\mathrm{i}}\left(\mathrm{k}_{\mathrm{i}}-1\right) / 2}
$$

Succinctly, $\mathrm{C}_{4 \mathrm{~b}}$ (i) is the probability that two neighbors of species $i$ share another neighbor in addition to $i$.

3) Are clusters formed by strong or weak interactions? Are we over or underestimating clustering coefficient of networks when ignoring link weight? If quadrilaterals are formed by strong interactions between species, clusters will play a larger role in both community structure and dynamics than if they are formed by weak interactions. To be able to answer this question, we also introduce a weighted clustering coefficient for bipartite graphs, $\mathrm{C}_{4 \mathrm{~b}}^{\mathrm{w}}(\mathrm{i})$, which is a generalization of $\mathrm{C}_{4 \mathrm{~b}}(\mathrm{i})$. Figure 1 also shows how to calculate $\mathrm{C}_{4 \mathrm{~b}}^{\mathrm{w}}(\mathrm{i})$. In the weighted version, the number of quadrilaterals is multiplied by the average normalized weight of the two links connecting node $\mathrm{i}$. This expression is given by

$\mathrm{C}_{4 \mathrm{~b}}^{\mathrm{w}}(\mathrm{i})=\frac{\sum_{\mathrm{m}, \mathrm{n}} \mathrm{q}_{\mathrm{imn}}\left(\frac{\tilde{\mathrm{w}}_{\mathrm{im}}+\tilde{\mathrm{w}}_{\mathrm{in}}}{2}\right)}{\mathrm{k}_{\mathrm{i}}^{\mathrm{nn}} \times \mathrm{k}_{\mathrm{i}}\left(\mathrm{k}_{\mathrm{i}}-1\right) / 2}$

where $\mathrm{q}_{\mathrm{imn}}$ is the number of quadrilaterals formed with nearest neighbors $m$ and $n$, and $\widetilde{w}_{i m}$ and $\widetilde{w}_{\text {in }}$ are the normalized weights of the links between $i$ and $m$ and between $i$ and $n$, respectively; where

$$
\tilde{\mathrm{w}}_{\mathrm{im}}=\frac{\mathrm{w}_{\mathrm{im}}}{\overline{\mathrm{w}_{\mathrm{i}}}}=\frac{\mathrm{w}_{\mathrm{im}}}{\left(\frac{s_{\mathrm{i}}}{\mathrm{k}_{\mathrm{i}}}\right)}
$$

Normalizing the weights ensures that the clustering coefficient will take a value between 0 and 1 .

To compare between binary and weighted networks, we will calculate the average clustering coefficient $\overline{\mathrm{C}_{4 \mathrm{~b}}}$ and the average weighted clustering coefficient $\overline{\mathrm{C}_{4 \mathrm{~b}}^{\mathrm{w}}}$. If the weighted clustering is larger than the binary one, $\overline{\mathrm{C}_{4 \mathrm{~b}}^{\mathrm{w}}} /$ $\overline{\mathrm{C}_{4 \mathrm{~b}}}>1$, species form highly interdependent clusters. On the other hand, if weighted clustering is smaller, clusters are formed by links with low weight and will have a minor 

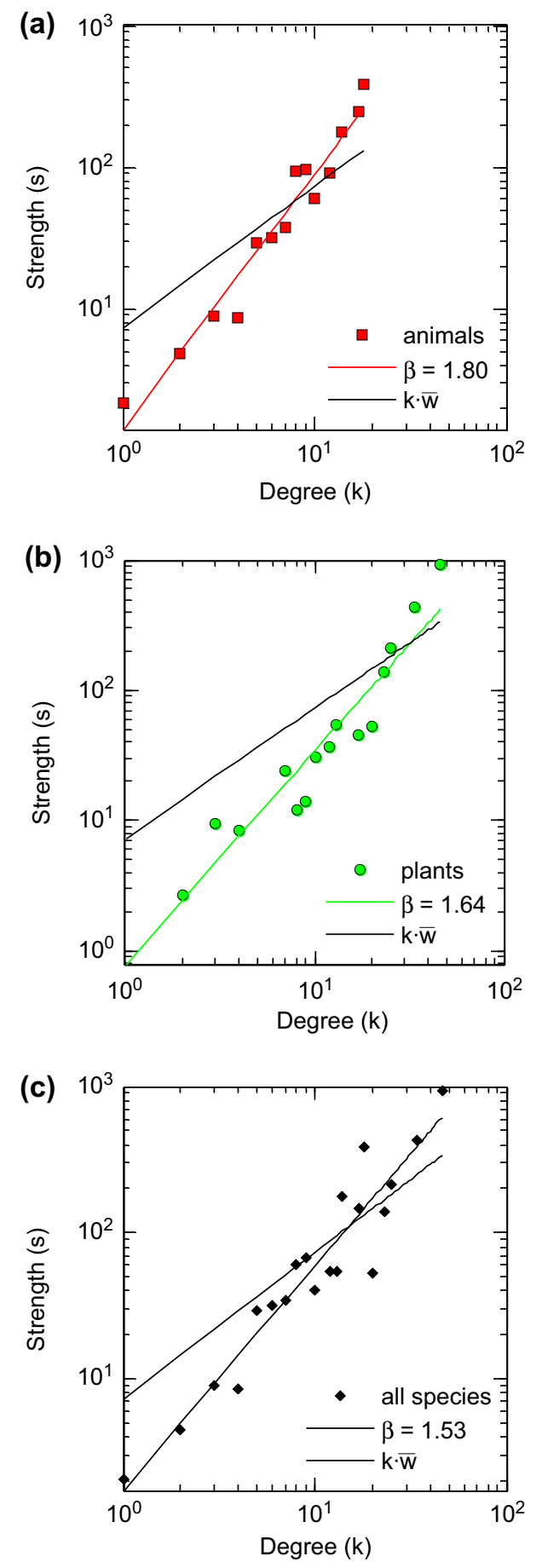

Figure 3. Correlation between species' strength and degree for the network of Memmott (1999). The solid line represents the best fit to the real data, and the dashed line represents the expected strength if weights were randomly assigned. Symbols represent the average node strength of all nodes with a certain degree. (a) Red squares represent data for animals. (b) Green circles represent data for plants. (c) Black diamonds represent the data for all species together. For randomized networks in which strength of a node is directly proportional to its degree, the exponent $\beta=1$. In contrast, the real data is best fit with an exponent $\beta$ significantly larger than one.

effect on the network structure. If $\overline{\mathrm{C}_{4 \mathrm{~b}}^{\mathrm{w}}} / \overline{\mathrm{C}_{4 \mathrm{~b}}} \approx 1$, binary clustering is a good measure for clustering in mutualistic networks, and incorporating link weights as we have in $C_{4 b}^{w}$ is unnecessary to understand the clustering phenomenon.
4) Will generalist species tend to be more or less clustered than specialist ones? Does this pattern change, when considering the weighted clustering coefficient? To address these questions, we compute the average (unweighted and weighted) clustering coefficient of species with the same degree $\mathrm{k}$. We then plotted the $\mathrm{C}_{4 \mathrm{~b}}(\mathrm{k})$ and the $\mathrm{C}_{4 \mathrm{~b}}^{\mathrm{w}}(\mathrm{k})$. These measures provide information about the small-world properties of mutualistic networks (Olesen et al. 2006).

5) Do species tend to interact with species that have a similar number of links than themselves? In mutualistic networks, it is known that specialists tend to interact with generalists (Vázquez and Aizen 2004). This phenomenon is called disassortative mixing (Newman 2002). Will this pattern be enhanced or disfigured by the consideration of link weights? To answer this question, we compare the average nearest-neighbors' degree, i.e. the average degree of all nodes linked to node $\mathrm{i}$, with the weighted average-nearest neighbors' degree introduced by Barrat et al. (2004) as

$\mathrm{k}_{\mathrm{nn}}^{\mathrm{w}}(\mathrm{i})=\frac{1}{\mathrm{~s}_{\mathrm{i}}} \sum_{\mathrm{j}=1}^{\mathrm{N}} \mathrm{w}_{\mathrm{ij}} \times \mathrm{k}_{\mathrm{j}}$

In Fig. A2 of the Supplementary material Appendix 1, we visually illustrate a case study to help calculate unweighted and weighted average-nearest neighbor's degree.

The origin and interpretation of this metric is as follows. Since interaction weights are the number of recorded interactions, we can reinterpret the weighted graph as a multigraph (Newman 2004) in which every visit, is now a link, and thus a plant-animal pair can share multiple links. By doing so, we consider that every recorded visit is a link between the two species. If the strongest interactions of a species occur with their most connected neighbors, $\mathrm{k}_{\mathrm{nn}}^{\mathrm{w}}(\mathrm{i})$ will be higher than $\mathrm{k}_{\mathrm{nn}}(\mathrm{i})$, and vice versa.

\section{Results}

If we focus on node-level properties, we find that the distribution of species' strength is as heterogeneous as the well known species' degree. Throughout the manuscript and remaining figures, we have used a plant pollinator network from Memmott (1999) as a representative example. In this example, the cumulative distributions of node degree and node strength are fitted to truncated-power laws (Fig. 2). The numerical values for every dataset are summarized in Supplementary material Appendix 1.

We have studied the relationship between the species' strength and its number of interactions in order to assess the shape of that correlation (Fig. 3). The dashed line represents the fit to a randomized network, where a node's strength is directly proportional to its degree and then exponent $\beta$ is equal to one. In contrast, fit to real data, solid line, exhibits a $\beta$ exponent significantly larger than one. Indeed, the value of the exponent $\beta$ is greater than 1 for the largest networks (Supplementary material Appendix 1 Table A2). The observed values of $\beta$ exponent demonstrate a non-linear relationship between species degree and species strength. It can be seen qualitatively as the difference between the slopes of the fits in Fig. 3. This result implies that highly-connected species have stronger interactions than expected if interaction weights are randomly distributed. Removing highly-connected 
(a)

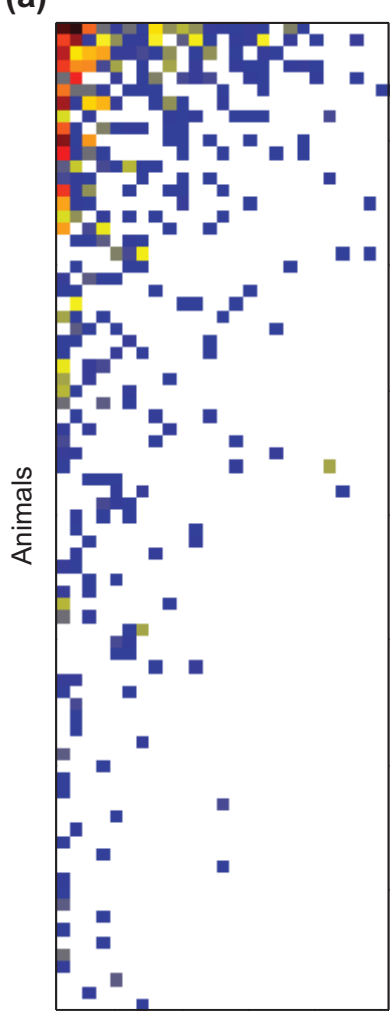

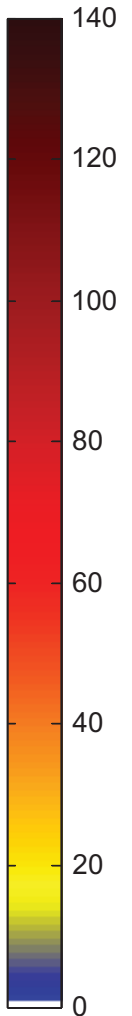

(b)

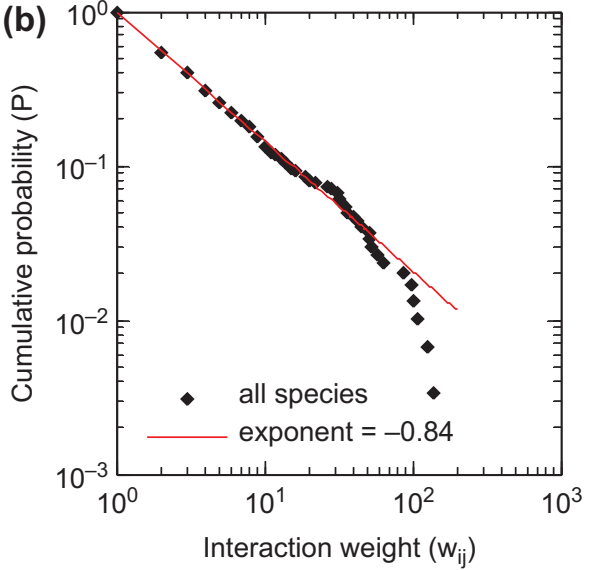

Figure 4. (a) Color plot of the weighted interaction matrix of Memmott (1999). Pollinators are represented in rows and plants in columns. We sort species by their degree such that species with the largest number of interactions are located in the top left corner. The color bar on the right corresponds to link weight (frequency of visits); white means no interaction is observed. (b) Interaction weight distribution, i.e. the cumulative probability of finding a link with a certain weight. The solid line is the fit to a power law with scaling exponent equal to $-0.81 \pm 0.02$. This power law fit holds for the majority of networks studied here.

species, therefore, has a quantitatively greater effect on a network than would be predicted solely by their degrees.

If we shift our focus from the nodes to their links, we find that the interactions between species in a mutualistic community are not equally weighted. Figure 4 a graphically shows how link weights are allocated in the network. As can be seen in the color bar, some interactions can be orders of magnitude larger than others from the same network. In fact, as is shown in Fig. $4 \mathrm{~b}$, the link-weight distribution follows a power-law. Table A3 in Supplementary material Appendix 1 shows the parameters of the fit to a power-law or a truncated power-law for the cumulative probability distribution of finding a link with a certain weight. Most interactions are weak whereas a few interactions are very strong.

The weighted interaction matrix depicted in Fig. 4a suggests that the strongest interactions occur between generalists (dark red color in the top-left corner). To quantify this pattern, we have studied the average weight as a function of end-point degree. We find a positive, significant relationship between the average weight of a link and the degree of the nodes that are connected by that link. This relationship is quantified by the exponent $\theta$. In Fig. 5, we plot the average weight of a link as a function of end-point degree for the same network described before. Since the exponent $\theta$ is larger than zero, we observe that the strongest interactions occur between high-degree nodes. Therefore, the larger the end-point degree of a link, the stronger the interaction between the two species connected by that link. Exponent $\theta$ is larger than zero for all networks studied with more than forty species. Supplementary material Table A4 shows the values of the exponent $\theta$ for all networks studied.

When considering binary information, generalists interact preferentially with specialists: the average degree of nearest neighbors $\mathrm{k}_{\mathrm{nn}}$ (i) decreases as node degree increases (Fig. 6). Therefore, nodes with high degree have a larger probability to be connected to low-degree nodes. This pattern is called disassortative mixing. Figure 6 shows that, when considering weighted links, disassortative mixing diminishes since the slope of the weighted data is less than that for the binary data. This occurs because a node's links with larger weights tend to point to neighbors with larger degree, in order for $\mathrm{k}_{\mathrm{nn}}^{\mathrm{w}}(\mathrm{i})>\mathrm{k}_{\mathrm{nn}}$ (i). As can be seen in Fig. 6, this difference is larger for highly-connected nodes. This means that, from the collection of a generalist's interactions, most are with specialists. Nevertheless, the weights are not equally distributed among these interactions. Interactions with specialists are weak whereas those few interactions with generalists are very strong, as was seen in Fig. 4a. All of the mutualistic networks studied exhibit similar results, as shown in Supplementary material Table A5.

We also observe differences between weighted and binary data when calculating the clustering coefficient. Figure 7 


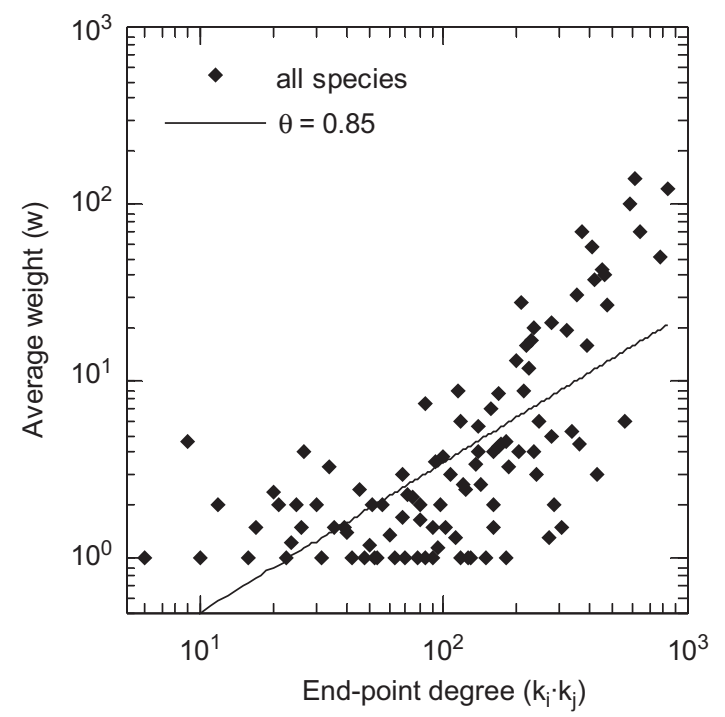

Figure 5. Average weight of links as a function of their end-point degree for the network of Memmott (1999). The solid line represents the best fit to the real data. The slope of this line is given by the exponent $\theta$. If the exponent $\theta$ is larger than zero, there is a positive correlation between the weight of an interaction and the product of interacting nodes' degrees. For the data displayed, $\theta=0.85 \pm 0.17$.

shows the difference between weighted and binary clustering coefficients distributed across species with different degree. We find that weighted clustering tends to be larger than binary clustering across the range of degrees. Moreover the difference between weighted and binary clustering is larger the larger the degrees (see also Fig. A1 in Supplementary material Appendix 1 to see more clearly how this difference grows). This result indicates that, in mutualistic networks, clusters tend to be formed by strong interactions. Indeed, the strongest interactions are between interconnected groups of generalist species. For a majority networks studied here, $\overline{\mathrm{C}_{4 \mathrm{~b}}^{\mathrm{w}}} / \overline{\mathrm{C}_{4 \mathrm{~b}}}>1$ for every degree.

\section{Discussion and conclusions}

From our examples, it is possible to appreciate the intricate relationship between weight and topology when characterizing mutualistic networks. We show how interaction weights are distributed across the skeleton provided by binary links. Our analysis of weighted networks provides novel information that cannot be obtained by measures based on topological information alone. It also allows us to directly compare weighted and unweighted metrics, and to explore the extent to which interaction weights are necessary to accurately characterize the organization of mutualistic communities. The present manuscript therefore compliments previous approaches at understanding the complex architecture of mutualistic networks.

One of the differences that we observe between binary and weighted mutualistic networks relates to the interpretation and quantification of species importance. If one species goes extinct, such a perturbation affects the whole network in terms of the loss of connectivity and the subsequent (a)

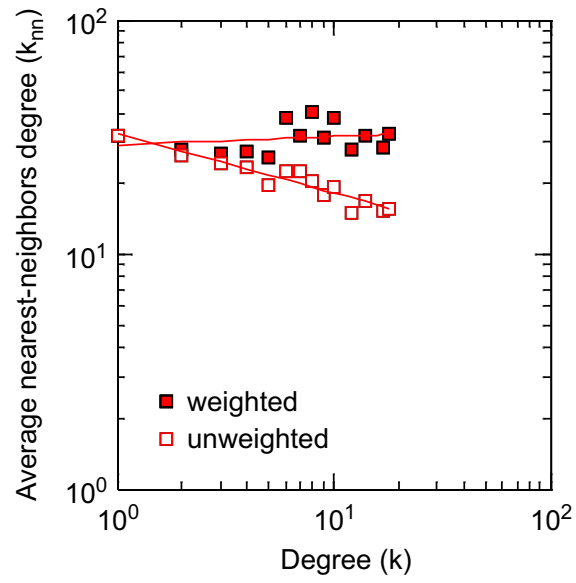

(b)

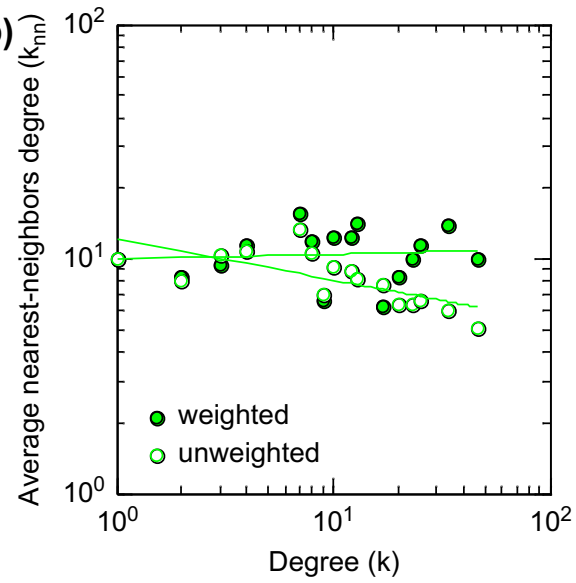

(c)

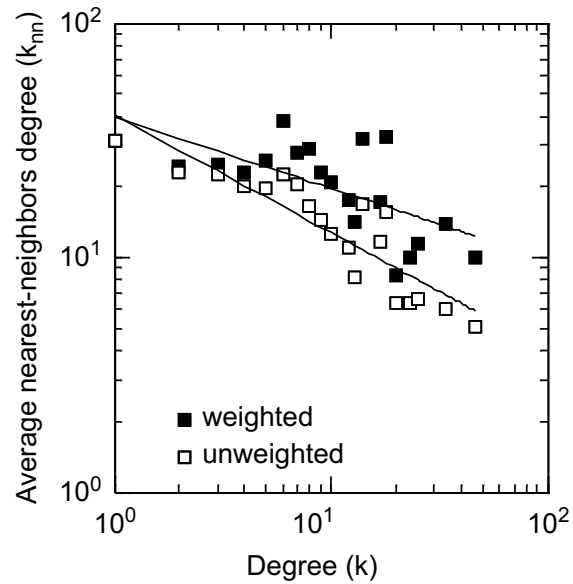

Figure 6. Average degree of nearest neighbors, $\mathrm{k}_{\mathrm{nn}}$, as a function of node degree for the network of Memmott (1999). Symbols represent the mean average-degree-of-nearest-neighbors for all nodes with a certain degree. Filled and open symbols correspond to weighted and binary data, respectively. The solid line is the best fit for the weighted network whereas the dashed line represents the best fit for the binary network. (a) Red squares represent data for animals. (b) Green circles represent data for plants. (c) Black diamonds represent the data for all species together. The negative slope, which represents dissasortativeness of unweighted data, tends to diminish or vanish for weighted data.

secondary extinctions (Memmott et al. 2004). The number of secondary extinctions after species extinction depends on the network connectivity and on the number of links of the species that goes extinct. Therefore, using binary 

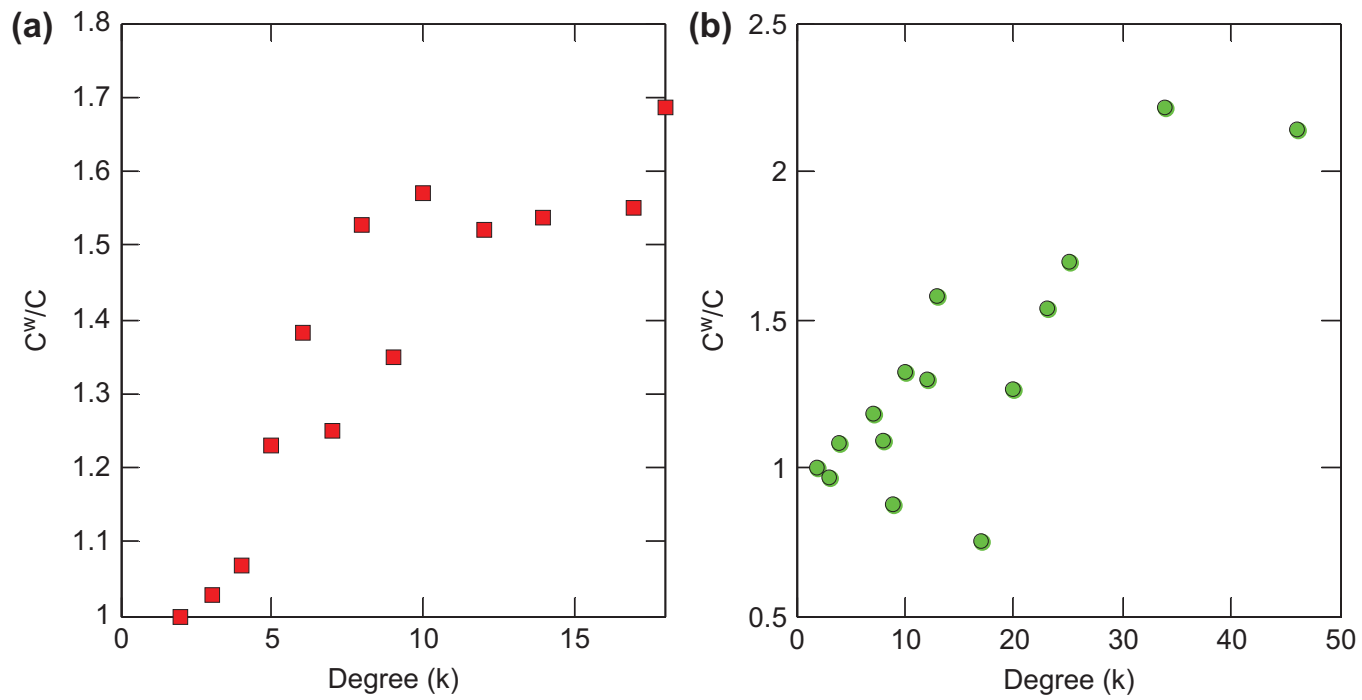

Figure 7. Distribution of average weighted clustering coefficient divided by average unweighted clustering coefficient for nodes with the same degree. (a) Red squares represent data taken from animals. (b) Green circles represent data taken from plants. For both animals and plants, species with larger degree have a larger weighted clustering coefficient relative to their binary clustering coefficient. For animals, the difference between weighted and binary coefficients is clearer due to better statistical power, since there are 79 animals compared to only 25 plants.

information, we can answer the question of how important a species is when preserving connectivity. Nevertheless, to measure energy flow through the network, link weights are necessary. By using interaction strength, we can answer the question of how important the same species is when preserving the total number of visits (Kaiser-Bunbury et al. 2010), other surrogates for interaction strength, or more precise measures that take into account pollination effectiveness. If the sole objective is to rank species according to their importance, weighted links are unnecessary in mutualistic networks (Scotti et al. 2007). Here, however, we show that, when compared to the rest of species in the network, highly-connected species are more important when preserving the total number of visits than they are when preserving network connectivity. Just as Bascompte and coworkers pointed out (Bascompte et al. 2006), species strength increasing faster than species degree implies that degree is insufficient to assess the importance of a species in a network.

With regard to species interactions, generalists tend to interact with specialists, and vice versa. This is manifested as the negative slope in the average nearest-neighbor's degree for the binary data: the larger a node degree, the lower the average degree of their neighbors (consistent with the observation by Vázquez and Aizen 2004). By comparing the observed pattern in binary networks to the same metric in weighted networks, we show that a species' strongest interactions occur with their most connected neighbors, and this tendency is larger the larger the degree of the species. Therefore, weighted and unweighted average nearest-neighbor's degree provide complementary information that can be used to answer different questions.

We also find that, while most interactions of generalists are with specialists, the strongest interactions occur between generalists themselves. As a result, generalists form not only interconnected groups of species, but strongly-interconnected groups of species. This pattern is not absent of evolutionary consequences. The presence of strong clusters between highly-connected species reinforces the idea of generalist species governing coevolution of the whole community (Thompson 2005). In mutualistic networks, convergence and complementarity of traits favor the incorporation of new species, resulting in a coevolutionary vortex that leads to the inclusion of even more species in the network of interactions (Thompson 2006). When a new species is included in the network, some of the species that were there previously obtain more interactions. If a certain species develops more interactions along evolutionary time, the strength of that species' interactions will also increase, and be redistributed across neighbors. That species will have a progressive tendency to tightly interact with other generalists, and that tendency creates the aforementioned clusters formed by strong links between generalists. We have shown that differences between weighted and unweighted clustering coefficients are larger the larger species' degree. This result is consistent with the recently reported importance of super-generalists for the evolutionary dynamics of mutualistic interactions (Guimarães et al. 2011). In looking at how weighted and unweighted clustering coefficient changes between species with different number of connections, one can see a fingerprint of how the coevolutionary vortex shapes the weighted architecture of mutualistic networks.

Quantitative interactions help reveal relevant patterns in the structure of mutualistic networks, as the patterns described here. The tight relationship between structure and dynamics (Thébault and Fontaine 2010) implies that, by improving our understanding of mutualistic network structure, we directly improve our predictive power over population dynamics and network structure. As suggested by Van Veen et al. (2006) for food webs, quantitative data provide useful tools to think about structuring processes. 
Acknowledgements - The authors would like to thank Jordi Bascompte, Daniel B. Stouffer and Pedro Jordano for their helpful comments on the manuscript. We also thank Daniel B. Stouffer for revising the English. This work was funded by project PGUI of the Comunidad de Madrid MODELICO-CM/S2009ESP-1691 (JG and JMP) and by the European Heads of Research Councils, the European Science Foundation, and the EC Sixth Framework Program through a EURYI (European Young Investigator) Award to Jordi Bascompte (LJG).

\section{References}

Barrat, A. et al. 2004. The architecture of complex weighted networks. - Proc. Natl Acad. Sci. USA 101: 3747-3752.

Bascompte, J. and Jordano, P. 2007. Plant-animal mutualistic networks: the architecture of biodiversity. - Annu. Rev. Ecol. Evol. Syst. 101: 221-223.

Bascompte, J. et al. 2006. Asymmetric coevolutionary networks facilitate biodiversity maintenance. - Science 312: 431-433.

Bastolla, U. et al. 2009. The architecture of mutualistic networks minimizes competition and increases biodiversity. - Nature 458: $1018-1020$.

Blüthgen, N. et al. 2008. What do interaction network metrics tell us about specialization and biological traits. - Ecology 89: 3387-3399.

Dobson, A. et al. 2006. Habitat loss, trophic collapse and the decline of ecosystem services. - Ecology 87: 1915-1924.

Elton, C. 1927. Animal ecology. - Univ. of Chicago Press.

Guimarães Jr, P. R. et al. 2011. Evolution and coevolution in mutualistic networks. - Ecol. Lett. 14: 877-885.

Herrera, C. M. 1987. Components of pollinator 'quality': comparative analysis of a diverse insect assemblage. - Oikos 50: 79-90.

Herrera, C. M. 1989. Pollinator abundance, morphology, and flower visitation rate: analysis of the 'quantity' component in a plant-pollinator system. - Oecologia 80: 241-248.

Hwang, J. K. et al. 2009. Structure of mutualistic complex networks. - Complex Sci. 4: 954-959.

Jordano, P. 1987. Patterns of mutualistic interactions in pollination and seed dispersal: connectance, dependence asymetries and coevolution. - Am. Nat. 129: 657-677.

Jordano, P. et al. 2003. Invariant properties in coevolutionary networks of plant-animal interactions. - Ecol. Lett. 6: 6981.

Kaiser-Bunbury, C. N. et al. 2010. The robustness of pollination networks to the loss of species and interactions: a quantitative approach incorporating pollinator behaviour. - Ecol. Lett. 13: 442-452.

Lind, P. G. et al. 2005. Cycles and clustering in bipartite networks. - Phys. Rev. E 72: 056127.

Supplementary material (available online as Appendix O19592 at <www.oikosoffice.lu.se/appendix>). Appendix 1.
Memmott, J. 1999. The structure of plant-pollinator food web. - Ecol. Lett. 2: 276-280.

Memmott, J. et al. 2004. Tolerance of pollination networks to species extinctions. - Proc. R. Soc. B 271: 2605-2611.

Newman, M. E. J. 2002. Assortative mixing in networks. - Phys. Rev. Lett. 89: 208701.

Newman, M. E. J. 2004. Analysis of weighted networks. - Phys. Rev. E 70 (5 Pt 2), 056131.

Olesen, J. et al. 2006. The smallest of all worlds: pollination networks. - J. Theor. Biol. 240: 270-276.

Onody, R. and de Castro, P. 2004. Complex networks study of brazilian soccer players. - Phys. Rev. E 037103.1-4.

Pastor-Satorras, R. and Vespignani, A. 2001. Epidemic spreading in scale-free networks. - Phys. Rev. Lett. 86: 3200.

Rezende, E. et al. 2007. Non-random coextinctions in phylogenetically structured mutualistic networks. - Nature 448: 925-928.

Ribeiro Mello, M. A. et al. 2011. The modularity of seed dispersal: differences in structure and robustness between bat- and bird-fruit networks. - Oecologia 176: 131-140.

Schupp, E. W. et al. 2010. Seed dispersal effectiveness revisited: a conceptual review. - New Phytol. 188: 333-353.

Scotti, M. et al. 2007. Weighting, scale dependence and indirect effects in ecological networks: a comparative study. - Ecol. Complex. 4: 148-159.

Solé, R. and Montoya, J. 2001. Complexity and fragility in ecological networks. - Proc. R. Soc. B 268: 2039-2045.

Stouffer, D. and Bascompte, J. 2010. Understanding food-web persistence from local to global scales. - Ecol. Lett. 13: 154161.

Thébault, E. and Fontaine, C. 2010. Stability of ecological communities and the architecture of mutualistic and trophic networks. - Science 329: 853-856.

Thompson, J. 2005. The geographic mosaic of coevolution. - Univ. of Chicago Press.

Thompson, J. 2006. Mutualistic webs of species. - Science 312: 372.

Van Veen, F. J. F. et al. 2006. Apparent competition, quantitative food webs, and the structure of phytophagous insect communities. - Annu. Rev. Entomol. 51: 187-208.

Vázquez, D. P. and Aizen, M. A. 2004. Asymmetric specialization: a pervasive feature of plant-pollinator interactions. - Ecology 85: 1251-1257.

Vazquez, D. P. et al. 2005. Interaction frequency as a surrogate for the total effect of animal mutualists on plants. - Ecol. Lett. 8: 1088-1094.

Watts, D. J. and Strogatz, S. H. 1998. Collective dynamics of 'small-world' networks. - Nature 393: 440-442.

Waser, N. M. and Ollerton, J. 2006. Plant-pollinator interactions: from generalization to specialization. - Univ. of Chicago Press. 